\title{
Ammonolysed $\mathrm{LiNi}_{0.8} \mathrm{Co}_{0.15} \mathrm{Al}_{0.05} \mathrm{O}_{2}$ as a cathode material for $\mathrm{Li}$-ion batteries with improved rate capability
}

\author{
SUKEUN YOON \\ Division of Advanced Materials Engineering and Institute for Rare Metals, Kongju National University, Chungnam 31080 , \\ Republic of Korea \\ skyoon@kongju.ac.kr
}

MS received 13 September 2017; accepted 21 February 2018; published online 3 December 2018

\begin{abstract}
In this work, ammonolysed $\mathrm{LiNi}_{0.8} \mathrm{Co}_{0.15} \mathrm{Al}_{0.05} \mathrm{O}_{2}$ (NCA) prepared by co-precipitation and subsequent ammonolysis was investigated as a cathode material for Li-ion batteries with enhanced rate capability. Detailed structural and morphological property characterization demonstrated that ammonolysis results in the incorporation of a small amount of nitrogen into the surface layer of the afore-mentioned material. The electrochemical performances of NCA electrodes were measured by galvanostatic charging-discharging of the corresponding Li-ion cells, revealing that ammonolysed NCA exhibited higher capacity and rate capability than those of the pristine sample, i.e., after 20 cycles, the discharge capacity of the former equalled $176 \mathrm{~mA} \mathrm{~h} \mathrm{~g}^{-1}$ at a current density of $18.4 \mathrm{~mA} \mathrm{~g}^{-1}$, remaining as high as $107 \mathrm{~mA} \mathrm{~h} \mathrm{~g}^{-1}$ at a high current density of $1840 \mathrm{~mA} \mathrm{~g}^{-1}$. This improved performance was ascribed to ammonolysis-induced surface changes, which reduced cell polarization during cycling and enhanced the electrochemical stability and reaction kinetics of NCA electrodes.
\end{abstract}

Keywords. Li-ion battery; $\mathrm{LiNi}_{0.8} \mathrm{Co}_{0.15} \mathrm{Al}_{0.05} \mathrm{O}_{2}$; ammonolysis.

\section{Introduction}

The development of medium-large Li-ion batteries for electric vehicles (EVs) and stationary storage of renewable energy has attracted increased attention, necessitating the search for new low-cost and safe electrode materials to meet the high-energy and high-power requirements of these applications [1,2]. However, $\mathrm{LiCoO}_{2}$, currently used in commercial Li-ion batteries, still suffers from the high cost of cobalt, chemical instability and toxicity, requiring a suitable alternative to solve these problems [3]. Among the broad range of layered compounds, lithium-nickel-cobalt mixed oxides have been proposed as promising alternatives to $\mathrm{LiCoO}_{2}$ as they exhibit high energy density and are of low raw material cost. However, although these nickel-rich cathode materials are both structurally and thermodynamically more stable than $\mathrm{LiCoO}_{2}$, their use in practical Li-ion cells still raises safety concerns [4-6]. Consequently, this problem has been mitigated using two approaches, namely covering the surface of nickel-rich cathode particles by a layer of salts and oxides or doping with other guest metal ions. The first approach, involving coating with species such as $\mathrm{Li}_{2} \mathrm{CO}_{3}, \mathrm{TiO}_{2}, \mathrm{AlF}_{3}$ and $\mathrm{Li}_{2} \mathrm{ZrO}_{3}$, has been shown to enhance cell cyclability and reduce the extent of side reactions between the electrode and the electrolyte [7-11]. On the other hand, partial substitution of $\mathrm{Ni}$ by $\mathrm{Mg}$, Co or $\mathrm{Al}$ improves electrode structural stability due to the radii of $\mathrm{Co}^{3+}$ and $\mathrm{Al}^{3+}$ being smaller than that of $\mathrm{Ni}^{3+}$, with substitution by these ions causing $a$-axis shrinkage $[12,13]$. In addition, surface modification with dopants such as $\mathrm{F}, \mathrm{Cl}$ and other elements has also been identified as a promising performance-enhancing technique, frequently increasing structural stability and electrical conductivity by hindering electrolyte degradation and reducing impedance $[14,15]$. Specifically, surface $\mathrm{N}$-doping by treatment with ammonia has been shown to effectively improve the electrochemical performance of spinel and Li-rich oxide layer materials, which is attributed to the formation of surface and oxygen defects and the nitridation of the active electrode material surface [16-18].

In this work, we aimed to elucidate the effect of ammonolysis on the surface properties and structure of $\mathrm{LiNi}_{0.8} \mathrm{Co}_{0.15} \mathrm{Al}_{0.05} \mathrm{O}_{2}$ (NCA) cathode materials and determine whether it can improve $\mathrm{Li}$-ion reaction kinetics and result in stable capacity retention during cycling because the enhancement of specific power density in Li-ion batteries is a principal issue in developing better EVs.

\section{Experimental}

\subsection{Preparation of ammonolysed NCA}

The $\mathrm{Ni}_{0.8} \mathrm{Co}_{0.15} \mathrm{Al}_{0.05}(\mathrm{OH})_{2}$ precursor was prepared by co-precipitation. $\quad \mathrm{NiSO}_{4} \cdot 6 \mathrm{H}_{2} \mathrm{O} \quad(>98 \%$, Aldrich), $\mathrm{CoSO}_{4} \cdot 7 \mathrm{H}_{2} \mathrm{O}(>99 \%$, Aldrich $)$ and $\mathrm{Al}_{2}\left(\mathrm{SO}_{4}\right)_{3} \cdot x \mathrm{H}_{2} \mathrm{O}(>98 \%$, Aldrich) were co-dissolved in deionized (DI) water in required stoichiometric ratios to obtain a transparent solution, which was added dropwise to aqueous $\mathrm{KOH}$, and the 
$\mathrm{pH}$, temperature and stirring speed of the obtained mixture were adjusted to the desired values. The produced precipitate was filtered, washed with DI water and dried in a vacuum oven to obtain $\mathrm{Ni}_{0.8} \mathrm{Co}_{0.15} \mathrm{Al}_{0.05}(\mathrm{OH})_{2}$, which was subsequently mixed with $5 \%$ excess $\mathrm{LiOH} \cdot \mathrm{H}_{2} \mathrm{O}$ and calcined in air at $850^{\circ} \mathrm{C}$ for $12 \mathrm{~h}$ to produce powdered NCA. Finally, the assynthesized NCA powder was subjected to ammonolysis in a vertical quartz tube at a temperature of $350^{\circ} \mathrm{C}$ and an $\mathrm{NH}_{3}$ flow rate of $0.21 \mathrm{~min}^{-1}$ for $1 \mathrm{~h}$. For brevity, non-ammonolysed and ammonolysed samples are denoted as pristine and ammonolysed NCA, respectively.

\subsection{Characterization}

The crystal structures of the synthesized samples were investigated by X-ray diffraction (XRD; D8 Bruker X-ray diffractometer with a $\mathrm{CuK}_{\alpha}$ radiation source) and $\mathrm{X}$-ray photoelectron spectroscopy (XPS; Thermo MultiLab 2000 spectrometer with a monochromatic $\mathrm{AlK}_{\alpha}$ radiation source), whereas morphologies were probed by scanning electron microscopy (SEM; Tescan Mira LM). Electrodes for electrochemical evaluation were prepared by mixing active material powder (80 wt\%), carbon black (Super C65, $10 \mathrm{wt} \%$ ) as a conducting agent and poly(vinylidene fluoride) (10 wt $\%$ ) dissolved in $N$-methylpyrrolidone as a binder to form a slurry. This slurry was coated onto Al foil, which was subsequently pressed and dried at $120^{\circ} \mathrm{C}$ for $2 \mathrm{~h}$ in vacuum. CR2032-type coin cells were assembled using polypropylene as a separator, Li metal foil as counter and reference electrodes, and $1 \mathrm{M} \mathrm{LiPF}_{6}$ in ethylene carbonate/diethyl carbonate $(1: 1, \mathrm{v} / \mathrm{v})$ as an electrolyte. Galvanostatic cycling was performed at a constant current density of $18.4 \mathrm{~mA} \mathrm{~g}^{-1}(0.1 \mathrm{C})$ in a potential window of 4.3-3.0 V vs. $\mathrm{Li}^{+} / \mathrm{Li}$.

\section{Results and discussion}

Figure 1 shows the XRD patterns of pristine and ammonolysed NCA, revealing that the reflections of both samples could be indexed to the hexagonal structure of $\alpha-\mathrm{NaFeO}_{2}$ ( $R 3 m$ space group), with no impurity phases detected. Notably, the incorporation of nitrogen did not result in obvious XRD pattern change. The $I(003) / I(104)$ ratio was used to quantify the degree of cationic mixing in the Li layer, with values above 1.2 considered acceptable [14]. Thus, the $I(003) / I(104)$ ratio of 1.44 and clearly split (006)-(102) and (108)-(110) peaks observed for NCA indicate its excellent layered cation ordering.

SEM imaging (figure 2) revealed that NCA comprised aggregates with an average size of $\sim 250 \mathrm{~nm}$, with no apparent difference in morphology and grain size observed after ammonolysis.

NCA is a representative Ni-rich layered material that provides a suitable environment for the stable existence of $\mathrm{Co}^{3+}$ and $\mathrm{Al}^{3+}$ ions, with the $\mathrm{Ni}^{3+} / \mathrm{Ni}^{2+}$ valence state having a large effect on the electrochemical properties of

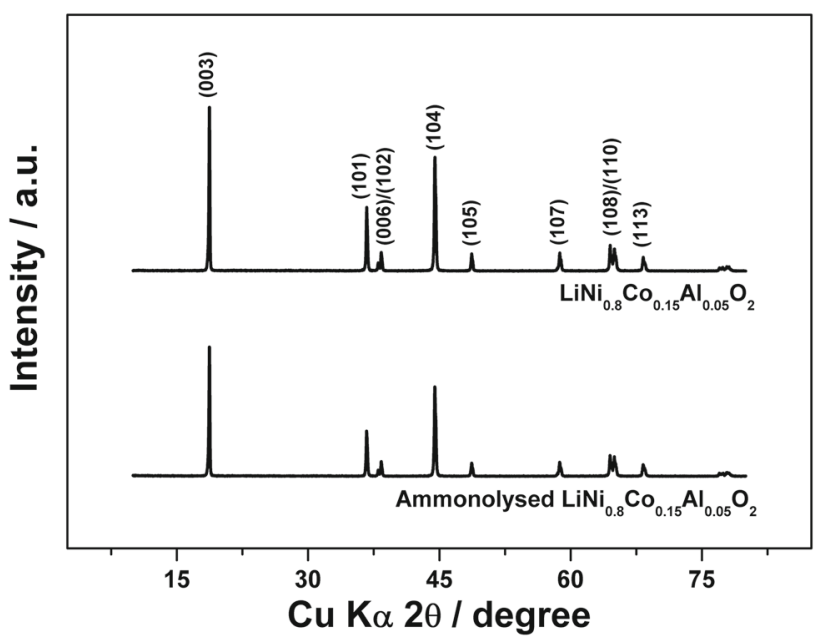

Figure 1. XRD patterns of pristine and ammonolysed NCA.

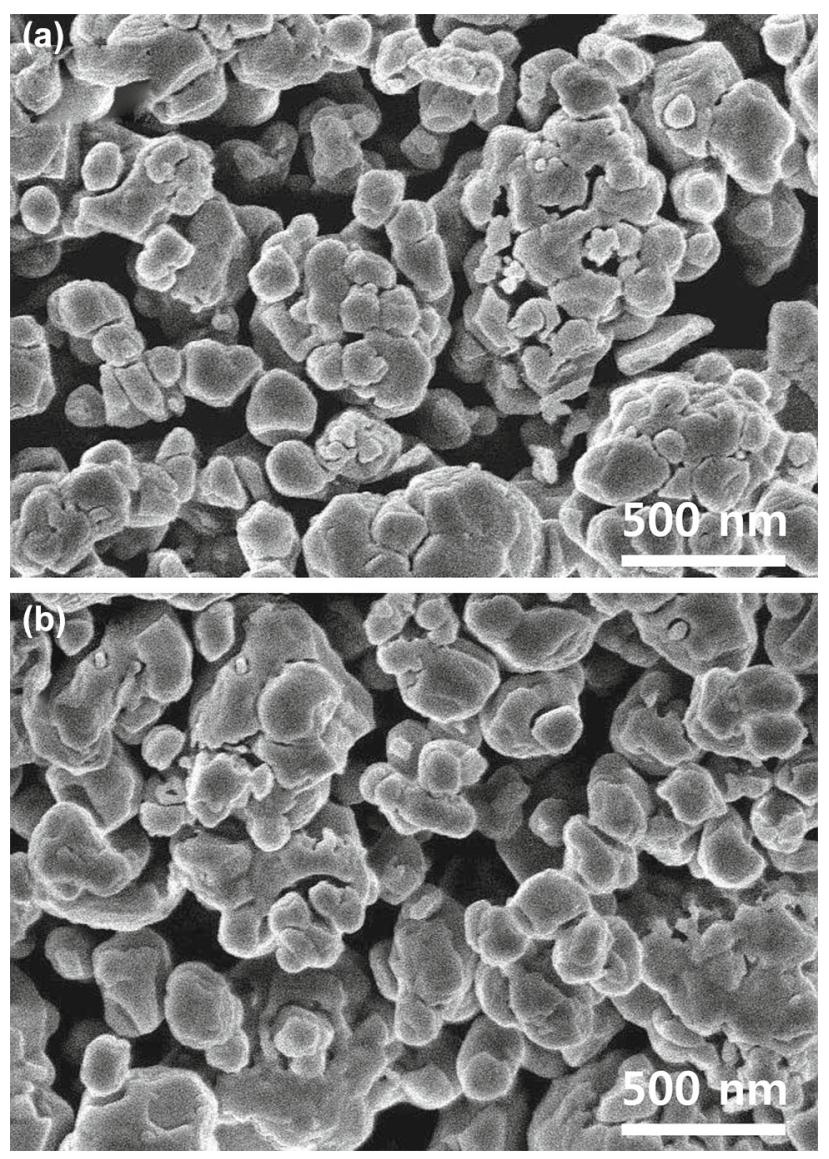

Figure 2. SEM images of (a) pristine and (b) ammonolysed NCA.

this material. Therefore, we utilized XPS to determine the oxidation state of transition metals and detect the presence of nitrogen, revealing the influence of surface modification on material properties. Figure 3 shows high-resolution Ni $2 \mathrm{p}$, Co $2 \mathrm{p}$ and $\mathrm{N}$ 1s XPS spectra of this cathode material, demonstrating that ammonolysis shifted the centre of the 

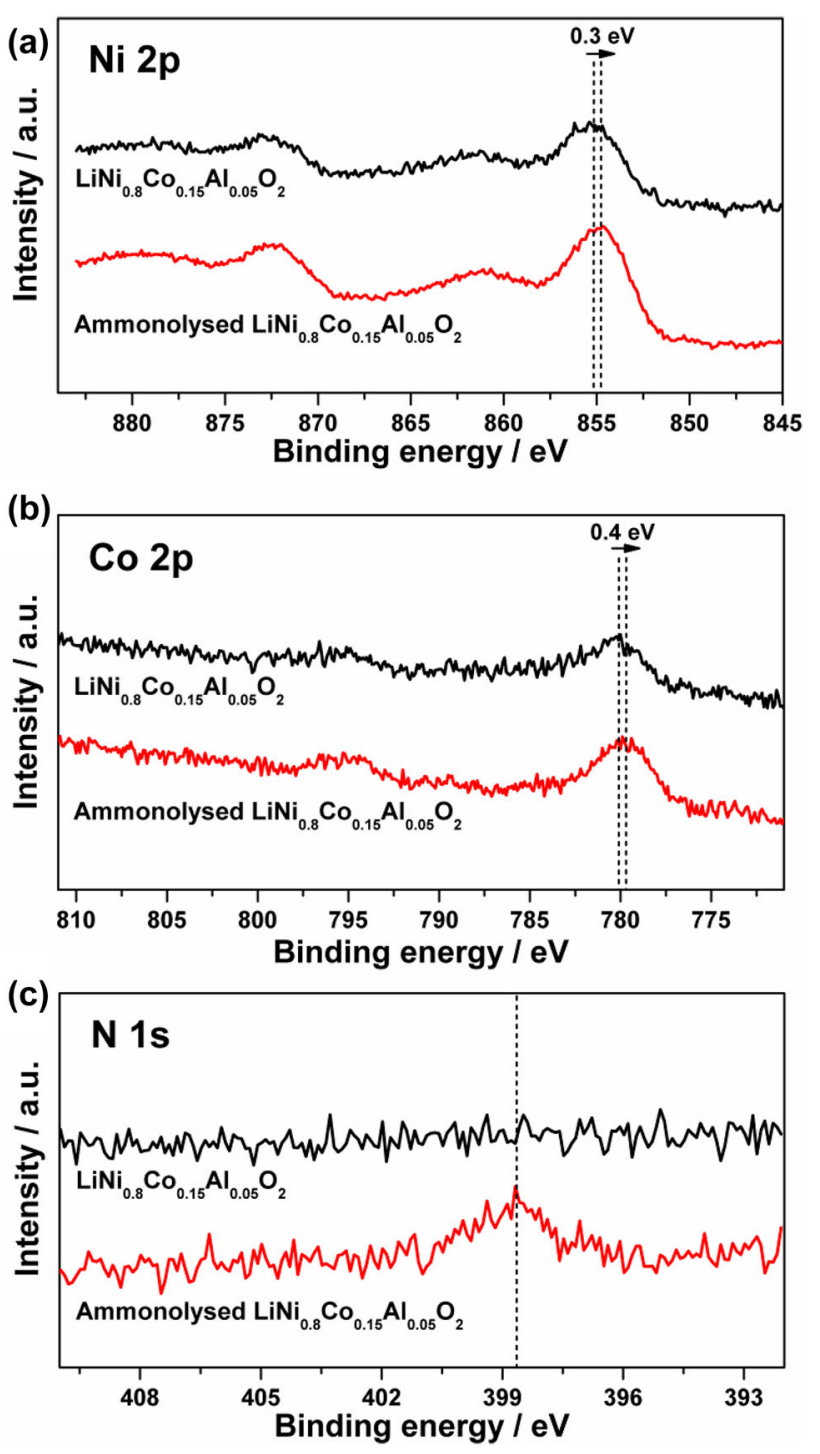

Figure 3. (a) Ni 2p, (b) Co $2 p$ and (c) N 1s XPS spectra of ammonolysed NCA.

Ni $2 p_{3 / 2}$ peak from 855.1 to $854.8 \mathrm{eV}$, while the Co $2 \mathrm{p}_{3 / 2}$ peak was shifted to lower binding energies (from 780.1 to $779.7 \mathrm{eV}$ ). Although these changes were attributed to the presence of elemental nitrogen in the surface layer of NCA [14,16-19], we could not correlate nickel or cobalt XPS data with nitrogen content due to the low magnitude of the latter parameter in ammonolysed NCA. However, the presence of the $\mathrm{N} 1 \mathrm{~s}$ peak at $398.6 \mathrm{eV}$ confirmed the presence of this element on the NCA surface.

The rate capability of Li-ion battery cathode materials is an important parameter for evaluating their potential for highpower applications, being related to Li-ion reaction kinetics and stable capacity retention. Figure 4 compares the rate capabilities of pristine and ammonolysed NCA after 2 and 20 cycles at various C-rates. At $0.1 \mathrm{C}\left(18.4 \mathrm{~mA} \mathrm{~g}^{-1}\right)$, both samples exhibited similar discharge capacities of $\sim 182$
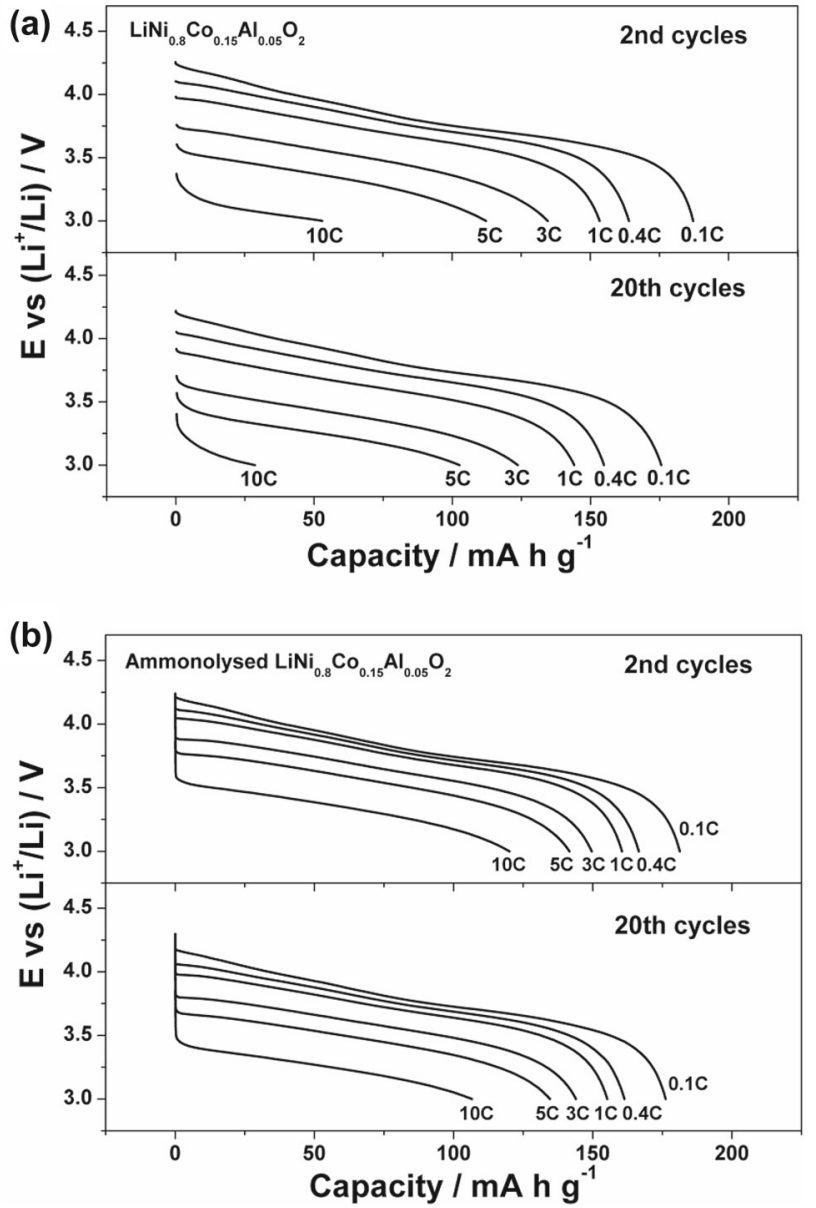

Figure 4. Discharge profiles illustrating the rate capabilities of (a) pristine and (b) ammonolysed NCA in the 2nd and 20th cycles.

(second cycle) and $175 \mathrm{~mA} \mathrm{~h} \mathrm{~g}^{-1}$ (20th cycle), indicating excellent capacity retention at low current density. However, pristine NCA exhibited low capacity retention after 2 and 20 cycles when cycling was performed at high Crates. Conversely, ammonolysed NCA featured a significantly higher rate capability, exhibiting discharge capacities of 120 (second cycle) and $107 \mathrm{~mA} \mathrm{~h} \mathrm{~g}{ }^{-1}$ (20th cycle) at $10 \mathrm{C}$ $\left(1840 \mathrm{~mA} \mathrm{~g}^{-1}\right)$. This result also indicates that it maintains a significantly high capacity retention at high current density compared with previously reported NCA particle without surface treatment $[20,21]$. Figure $5 \mathrm{a}$ and $\mathrm{b}$ shows the discharge capacities of pristine-ammonolysed NCA normalized with respect to those at $0.1 \mathrm{C}\left(18.4 \mathrm{~mA} \mathrm{~g}^{-1}\right)$ and obtained at various $\mathrm{C}$-rates in the second and 20th cycle, respectively. As mentioned earlier, ammonolysed NCA exhibited significantly better rate capabilities than pristine NCA in the 20th cycle. Furthermore, as shown in figure $5 \mathrm{c}$, rate capability retention is defined as the ability to maintain rate capability during cycling and calculated as the percentage ratio of the rate capability measured in the second cycle to that measured in the 20 th cycle. The difference in rate capability retention between 


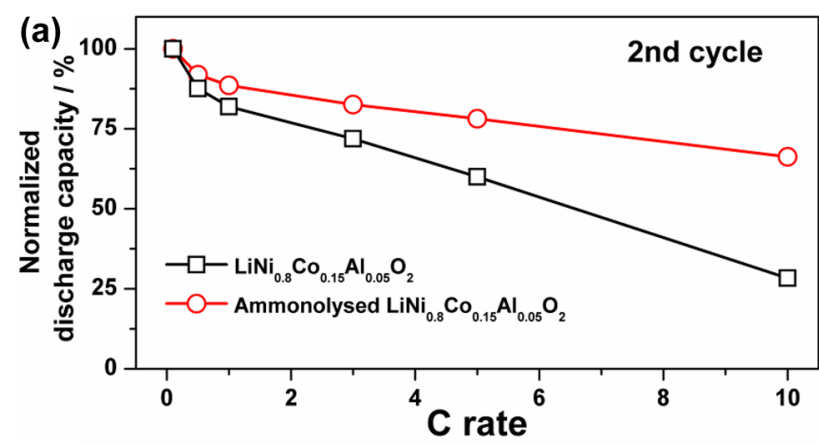

(b)

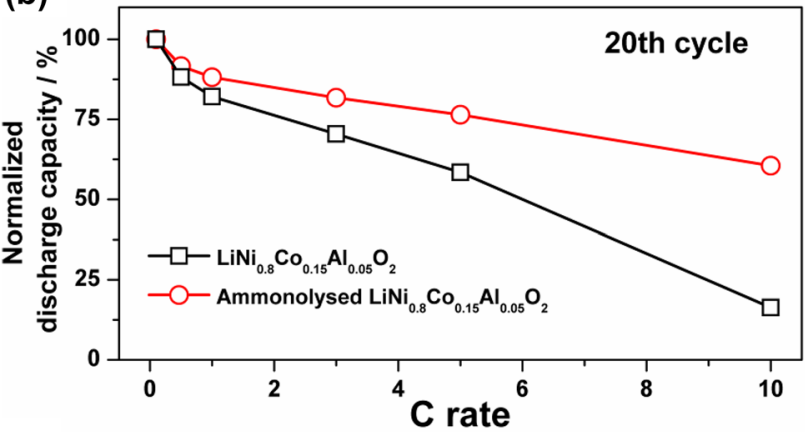

(c)

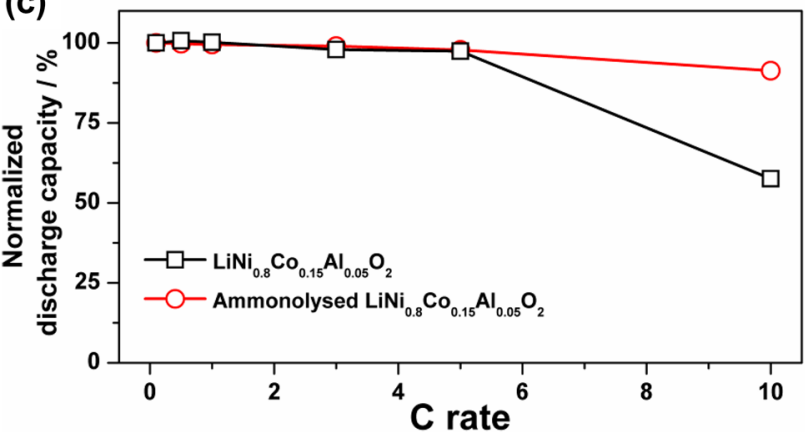

Figure 5. Comparison of rate capabilities and rate capability retentions of pristine and ammonolysed NCA. (a) Normalized discharge capacities in the second cycle, (b) normalized discharge capacities in the 20th cycle and (c) rate capability retentions.

pristine and the ammonolysed NCA increased after several cycles, implying that the latter sample featured a lower polarization than the former.

To understand the relationship between rate capability and total polarization resistance $\left(R_{\mathrm{p}}\right)$, the second-cycle discharge profiles of both samples were further analysed. Figure 6 shows linear voltage $v s$. mass current plots for depth of discharge (DOD) values from 10 to $50 \%$, with the total polarization resistances (including those of the cathode/anode, electrolyte and separator) deduced from the slope of these plots. Figure 7 shows $R_{\mathrm{p}} v s$. DOD plots for pristine and ammonolysed NCA in the second cycle, revealing that $R_{\mathrm{p}}$ decreased with increasing DOD (up to $50 \%$ ) and indicating that the discharge kinetics was favoured. Thus, these results indicated that the $R_{\mathrm{p}}$ of ammonolysed NCA was smaller than that of pristine NCA due to the increased surface chemical stability and electron conductivity of the former, as
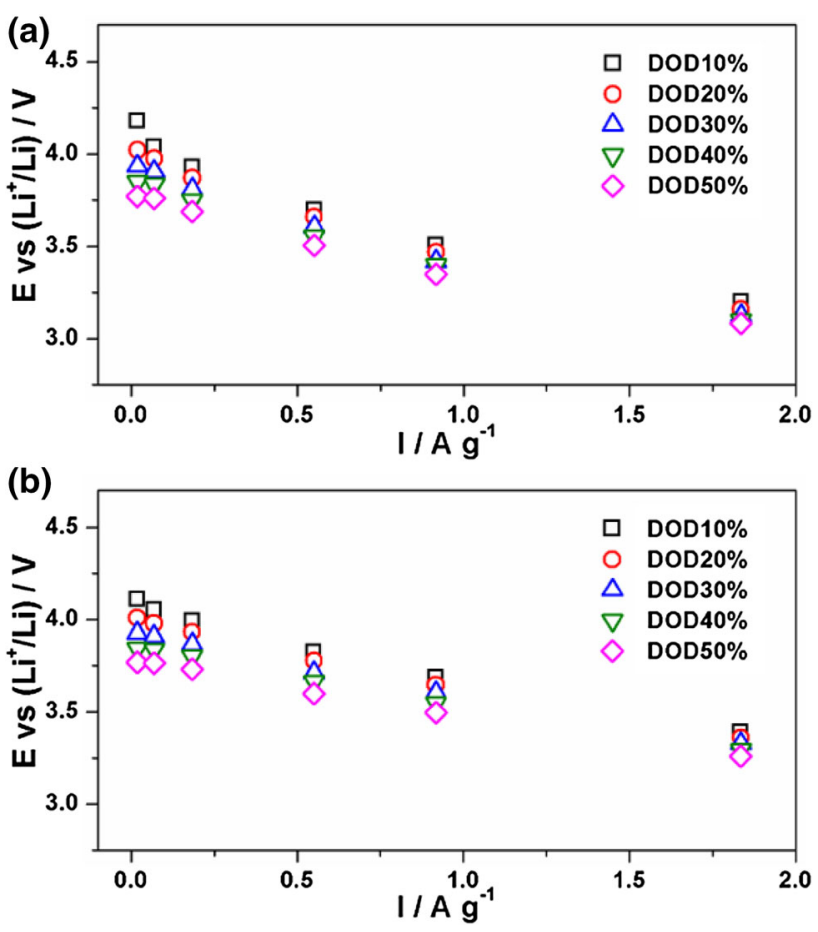

Figure 6. Relationship between potential and mass current of (a) pristine and (b) ammonolysed NCA in the second cycle at different DOD values.

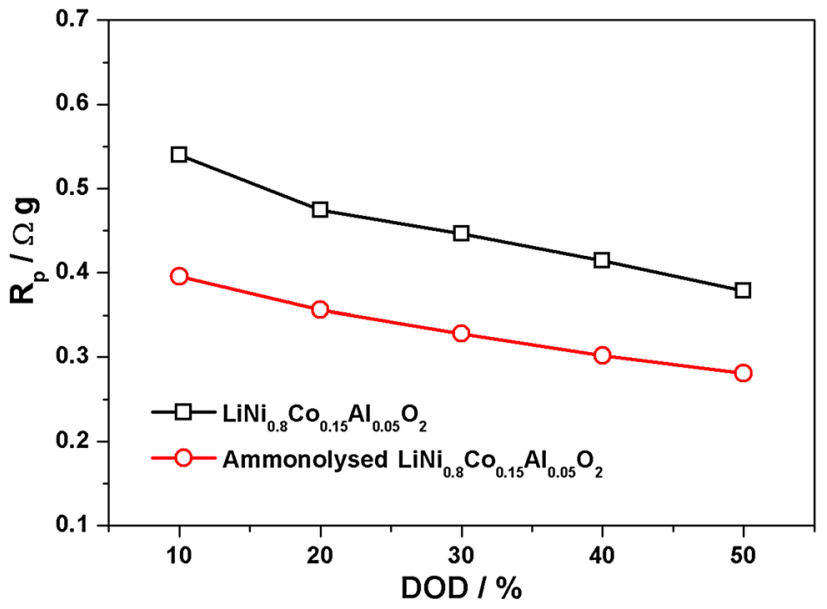

Figure 7. Dependence of polarization resistance $\left(R_{\mathrm{p}}\right)$ on DOD for pristine and ammonolysed NCA in the second cycle.

confirmed by the rate capability retention data in figure 6 . These enhanced electrochemical properties were ascribed to the effect of nitrogen doping, which resulted in structural stability, increasing electrical conductivity at the electrolytecathode interface [22,23]. Moreover, the incorporated nitrogen facilitated fast electron transport and acted as a channel for fast lithium ion diffusion, supporting the fast solvationdesolvation of these ions. Since nitrogen interacts with $\mathrm{Li}$ ions more strongly than the oxide anion, the presence of the 
former was expected to promote the $\mathrm{Li}$ ion transition at the interface between solvated $\mathrm{Li}$ ions in the electrolyte and the active material.

\section{Conclusion}

In this work, we successfully utilized ammonolysis to improve the electrochemical performance of an oxide layered cathode material for Li-ion batteries, successfully preparing ammonolysed NCA by co-precipitation and subsequent ammonolysis and demonstrating that surface nitrogen doping resulted in increased rate capability. After 20 cycles, ammonolysed NCA exhibited a discharge capacity of $107 \mathrm{~mA} \mathrm{~h} \mathrm{~g}^{-1}$ at $10 \mathrm{C}$, which corresponded to stable cycling and was nearly three times higher than that of pristine NCA. The nitrogen in ammonolysed NCA was shown to provide high electrical conductivity along the surface, thus enhancing rate capability and revealing that ammonolysis is a powerful method of improving the electrochemical performance of electrode materials for Li-ion batteries.

\section{Acknowledgements}

This research was supported by the Ministry of Trade, Industry and Energy (MOTIE), Korea Institute for Advancement of Technology (KIAT), through the Encouragement Program for the Industries of Economic Cooperation Region (No. 10067294), Korea Evaluation Institute of Industrial Technology (KEIT, No. 10067294) and the National Research Foundation of Korea (NRF, No. 2018R1C1B6004689).

\section{References}

[1] Dunn B, Kamath H and Tarascon J M 2011 Science 334928
[2] Polle B G, Staffell I and Shang J L 2012 Electrochim. Acta 84 235

[3] Tebbe J L, Holder A M and Musgrave C B 2015 ACS Appl. Mater. Interfaces 724265

[4] Kang K S, Choi S, Song J, Woo S G, Jo Y N, Choi J et al 2014 J. Power Sources 25348

[5] Wu F, Tian J, Su Y, Wang J, Zhang C, Bao L et al 2015 ACS Appl. Mater. Interfaces 77702

[6] Manthiram A, Knight J C, Myung S T, Oh S M and Sun Y K 2016 Adv. Energy Mater. 61501010

[7] Liu H, Zhang Z, Gong Z and Yang Y 2004 Solid State Ion. 166 317

[8] Li C, Zhang H P, Fu L J, Liu H, Wu Y P, Rahm E et al 2006 Electrochim. Acta $\mathbf{5 1} 3872$

[9] Lee S H, Yoon C S, Amine K and Sun Y K 2013 J. Power Sources 234201

[10] Liu W, Hu G, Du K, Peng Z and Cao Y 2013 Surf. Coat. Technol. 216267

[11] Song B, Li W, Oh S M and Manthiram A 2017 ACS Appl. Mater. Interfaces 99718

[12] Liu Q, Du K, Guo H, Peng Z D, Cao Y B and Hu G R 2013 Electrochim. Acta 90350

[13] Huang B, Lin X, Wang Z, Guo H and Xiong X 2014 Ceram. Int. 4013223

[14] Li X, Kang F, Shen W and Bai X 2007 Electrochim. Acta 53 1761

[15] Zhu L, Liu Y, Wu W, Wu X, Tang W and Wu Y 2015 J. Mater. Chem. A 315156

[16] Zhang H Z, Qiao Q Q, Li G R, Ye S H and Gao X P 2012 J. Mater. Chem. 2213104

[17] Li B, Li C, Cao Z, Wang J and Zhao J 2016 RSC Adv. 631014

[18] Erickson E M, Sclar H, Schipper F, Liu J, Tian R, Ghanty C et al 2017 Adv. Energy Mater. 71700708

[19] Son J T and Kim H G 2005 J. Power Sources 147 220

[20] Zhuang G V, Chen G, Shim J, Song X, Ross P N and Richardson T J 2004 J. Power Sources 134293

[21] Chen Y, Li P, Zhao S, Zhuang Y, Zhao S, Zhou Q et al 2017 RSC Adv. 729233

[22] Choi W and Manthiram A 2007 J. Electrochem. Soc. 154 A792

[23] Wu Y and Manthiram A 2009 Solid State Ion. 18050 